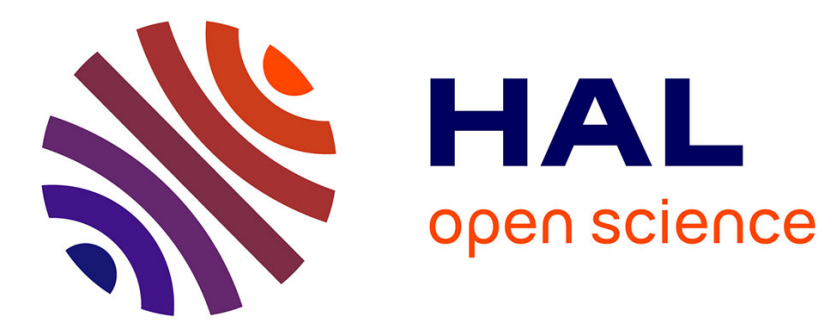

\title{
Development of a Product Configuration System for Cloud Manufacturing
}

\author{
Shiqiang Yu, Xun Xu
}

\section{To cite this version:}

Shiqiang Yu, Xun Xu. Development of a Product Configuration System for Cloud Manufacturing. IFIP International Conference on Advances in Production Management Systems (APMS), Sep 2015, Tokyo, Japan. pp.436-443, 10.1007/978-3-319-22759-7_51. hal-01431130

\section{HAL Id: hal-01431130 \\ https://hal.inria.fr/hal-01431130}

Submitted on 10 Jan 2017

HAL is a multi-disciplinary open access archive for the deposit and dissemination of scientific research documents, whether they are published or not. The documents may come from teaching and research institutions in France or abroad, or from public or private research centers.
L'archive ouverte pluridisciplinaire HAL, est destinée au dépôt et à la diffusion de documents scientifiques de niveau recherche, publiés ou non, émanant des établissements d'enseignement et de recherche français ou étrangers, des laboratoires publics ou privés. 


\title{
Development of a Product Configuration System for Cloud Manufacturing
}

\author{
Shiqiang $\mathrm{Yu}$ and $\mathrm{Xun} \mathrm{Xu}^{*}$ \\ Department of Mechanical Engineering, The University of Auckland, Auckland, New Zealand \\ syu431@aucklanduni.ac.nz and xun.xu@auckland.ac.nz
}

\begin{abstract}
For Configure-to-Order manufacturing firms, Product Configuration System (PCS) is one of a key components. PCS has been introduced for decades and its technology has matured, providing the industry with a set of usability standards and best practices. However, even the state-of-the-art PCS performs poorly in terms of connectivity, customisation freedom management and implementation. In this regard, this paper first reviews the impact of manufacturing paradigms on product customisation and take Cloud Manufacturing, a nascent manufacturing model, as a disruptive technology to surmount existing obstacles. Then, a general architecture of PCS for Cloud Manufacturing (CM-based PCS) is proposed to conduct configuration reasoning based on cloud-sourced configuration knowledge, to diversify configurable options by introducing proper Cloud Manufacturing services and to fulfil configuration orders through a cloud-based design and manufacturing platform.
\end{abstract}

Keywords: Product Configuration. Cloud Manufacturing. Everything-as-a-service. Ontology.

\section{Introduction}

With emerging disruptive technologies and changing of Customer Needs (CNs) and market demand, manufacturing industry has experienced several paradigms. Mass Customisation (MC) emerged from the 1980s, emphasising the need to provide outstanding service to customers in providing products that meet CNs at a low cost [1]. Due to trend of globalisation, paradigms complying with more specific CNs emerged, such as oneof-a-Kind Production (OKP) emphasising the "market-of-one", and Mass Personalization (MP) aiming to reveal latent CNs [2].

Recently, a new service-oriented manufacturing model, Cloud Manufacturing (CM), emerged. As a nascent paradigm, CM is considered as a promising paradigm that will change the way manufacturing businesses operate. In the literature, Cloud Manufacturing-related definitions have been elaborated by different researchers. Main stream definitions of CM contain key words like networked manufacturing, ubiquitous access, multi-tenancy and virtualization, big data and the IoT, everything-as-a-service (e.g.,

* Corresponding author. Tel.: +64 9373 7599. E-mail addresses: xun.xu@auckland.ac.nz. 
infrastructure-as-a-service, platform-as-a-service, hardware-as-a-service, and software-as-a-service), scalability, and resource pooling [3-5].

It is obvious that the evolvement of manufacturing paradigms is affecting the way of producing customised products. To achieve increased customisation requirement, manufacturers' resource and capability for design and manufacturing are met with challenges. Customisation freedom, to a certain degree, depends on the volume of manufacturing resources at hand, so new emerging manufacturing paradigms will boost indepth customisation.

Comparing to conventional manufacturing paradigms, Cloud Manufacturing makes manufacturing firms more accessible to external manufacturing resources. From this point of view, Cloud Manufacturing is a remedy bridging the gap between diverse CNs and insufficient internal manufacturing resources and capability. Obviously, manufacturing enterprises, in particular Small and Medium-sized Enterprises (SMEs), can deliver more in-depth customisation by adopting Cloud Manufacturing.

Product configuration is an activity of customising a product to meet the needs of a particular customer. Product Configuration System (PCS) is a tool to conduct an activity of customising a product to meet the needs of a particular customer. To utilise manufacturing resources in a "cloud", a new generation PCS is a necessity for configuration in a cloud environment.

\section{Challenges of Developing the Next Generation PCS}

\subsection{Issues of Existing PCS}

With the introduction of AI and cloud technologies, cloud-based PCS is currently the most advanced. Recently, numerous PCS vendors have launched their "cloud-based product configuration solutions", in which cloud concept is embedded.

In fact, those cloud-based PCSs in use are far from being perfect. Firstly, none of cloud-based PCSs could quickly respond to changes in the production systems and the supply chain. Such a drawback results in an array of issues like non-support of dynamically processing Request for Quotation (RFQ) and lower degrees of product customisation freedom to customer. Besides, the cost of the maintenance and management of configuration system is truly high especially when the configurator aims at structural and compositional configuration of a complex Engineering-to-Order (ETO) product. It is because any change of manufacturing capability or disruption of supply chain may incur momentous adjustment of configuration options and pricing/quote. Another factor restricting the application of PCS is that implementing a new PCS requires significant and potentially painful changes in the way the order acquisition and fulfilment activities are organised, and necessitate a high initial investment in terms of man-hours.

\subsection{Future of PCS}

Main stream ERP vendors (e.g. SAP and Infor), CAx software vendors (e.g. Autodesk) and some other software vendors (Tacton, KBMAX and Mycustomizer) have delivered their cloud-based product configuration systems. With features of PaaS and IaaS, these 
cloud-based solutions enable manufacturing firms to provide engineers, sales reps, and end customers with web and even mobile access to a configuration as a thin client (i.e. with nothing to install). Those cloud solutions only enhanced accessibility and computing capability of PCS, but made very little difference to above-mentioned gaps.

As mentioned in Section 1, phasing in Cloud Manufacturing will facilitate more indepth product customisation. Meanwhile, manufacturing resources and capability encapsulated as cloud services in Manufacturing Cloud is promising to revolutionise PCS in future. For instance, numerous manufacturing services could greatly increase the customisation freedom of products; an open architecture PCS platform integrated with Manufacturing Cloud is much easier for an enterprise to develop a brand new PCS or to upgrade dated one. As manufacturing services can be published in terms of real-time information, any changes happened in supply chain and outsourced supply chain are able to be taken into account in each configuration.

For those reasons, this research considers Cloud Manufacturing as a disruptive technology to upgrade its precedents and focuses on developing Product Configuration System for Cloud Manufacturing (abbreviated as CM-based PCS in following paper). In comparison with conventional cloud-based PCSs getting access to computing cloud, storage cloud and etc., CM-based PCSs further integrate with Manufacturing Cloud which encapsulated a wide range of manufacturing services stemmed from distributed manufacturing service providers. Such connectivity to manufacturing resources and capability makes CM-based PCS able to react to dynamic requirements from customers, and meanwhile enhances configurability of products by discovering new manufacturing services which can be applied to the development of new product variants.

\section{Implementation of CM-based PCS}

Nowadays, ever-increasing resources from the plant floor and headquarter are virtualised and encapsulated as cloud services, and next generation applications (e.g., PCS) definitely require connectivity to ubiquitous clouds. Integrating a PCS with Manufacturing Cloud will facilitate scalability and utilisation of facilities [6]. So far, there is no literature on any systematic methodology of implementing CM-based PCS.

\subsection{Integration with Manufacturing Cloud}

The research utilises the cloud computing concept and service-oriented principles to enhance conventional PCS technology. To achieve that, the concept of Manufacturingas-a-Service (MaaS) and Design-as-a-Service (DaaS) are embedded here. Based on the concept of MaaS, to fulfil product configuration, production systems on the basis of accessibility of PCS can be integrated with manufacturing services via a certain Cloudbased Design and Manufacturing (CBDM) platform [7]. It provides users with a level of product customisation freedom that is directly integrated to the available manufacturing capabilities of production sites within a cloud manufacturing infrastructure. Meanwhile, manufacturing services stemmed from the visualisation of pool of manu- 
facturing resource are promising to boost the capability of product design within configuration process by creating a dynamic co-design environment for the interaction between customers and manufacturers.

Manufacturing services may be internal (i.e. the manufacturer itself) or external (i.e. supplier, outsourced supplier, etc.). Manufacturing Cloud can be classified similarly by introducing the concept of cloud computing embracing four types of deployment models, including 1) private cloud: a centralised management effort in which manufacturing services are shared within one company or its subsidiaries; 2) community cloud: a collaborative effort in which manufacturing services are shared between several organisations from a specific community with common concerns; 3 ) public cloud: a set of manufacturing services in which manufacturing services are available to the general public [7]. To seek proper manufacturing resource, manufacturing firms would typically give priority to their internal manufacturing resources, then, if necessary, cooperation within community would be another good choice, and an anonymous supplier from public cloud will be the last option.

\subsection{Architecture of the Reasoning Engine}

Considering incremental changes and updates on products due to new technology advances and the expansion of the Internet, a configuration model supports knowledge reuse and sharing is crucial. In order to utilise ubiquitous cloud and realise configuration based on CM, an ontology-based approach is adopted in this research.

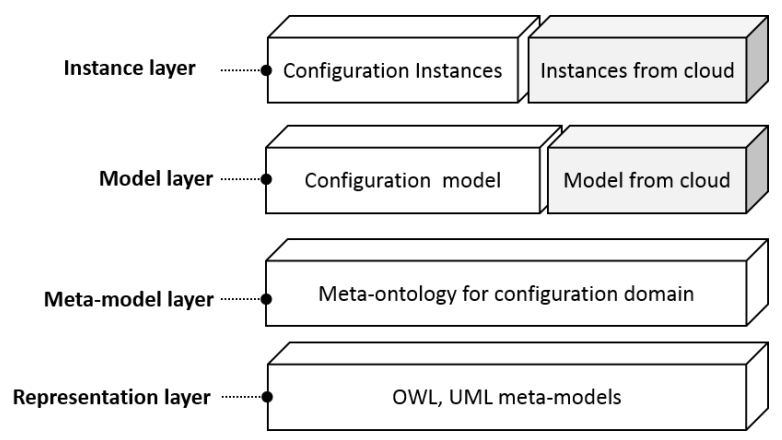

Fig. 1. Four-layer modelling architecture

Ontology is a formal, explicit specification of a shared conceptualisation. There has been literature of addressing the modelling of product configuration knowledge with an ontology-based approach in which structural knowledge is formalized in OWL (Ontology Web Language) [8] and constraint knowledge in SWRL [9], (Semantic Web Rule Language). Through the transformation of configuration knowledge into Jess facts and Jess rules, actual configuration processes are carried out with the support of Jess [10], a rule engine for the Java platform.

To encourage reuse of configuration models and flexibility in representing knowledge, the presented modelling approach for product configuration knowledge follows four-layer architectures [11]. Unlike a usual ontology-based configuration engine, 
the instance layer and the model layer are integrated cloud-sourced knowledge for extension in this research (see Fig. 1). The difficulties in modelling product configuration knowledge can be dramatically reduced by constructing configuration models based on existing configuration knowledge instead of starting for scratch.

The advantage of OWL-based configuration models is that the reuse of configuration models can be ensured since OWL, as an ontology language, supports knowledge reuse and sharing. This is especially crucial, considering incremental changes and updates on products due to new technology advances.

\subsection{Prototype of the CM-based PCS}

To integrate the ontology-based PCS with Manufacturing Cloud, we propose a novel way to obtain constraint knowledge in SWRL by extracting and parsing distributed configuration knowledge stemmed from the resource pool of manufacturing resources. The thorny problems here include: 1) to establish a communication mechanism enabling the interaction between PCS and Manufacturing Cloud; 2) to depict configuration-relevant manufacturing resources and external inventory information in a unified way which supports the reasoning of Ontology-based PCS engine; 3) to archive CMbased request for manufacturing (RFM), including production documentation and product information model, in an easily accessible and retrievable manner.

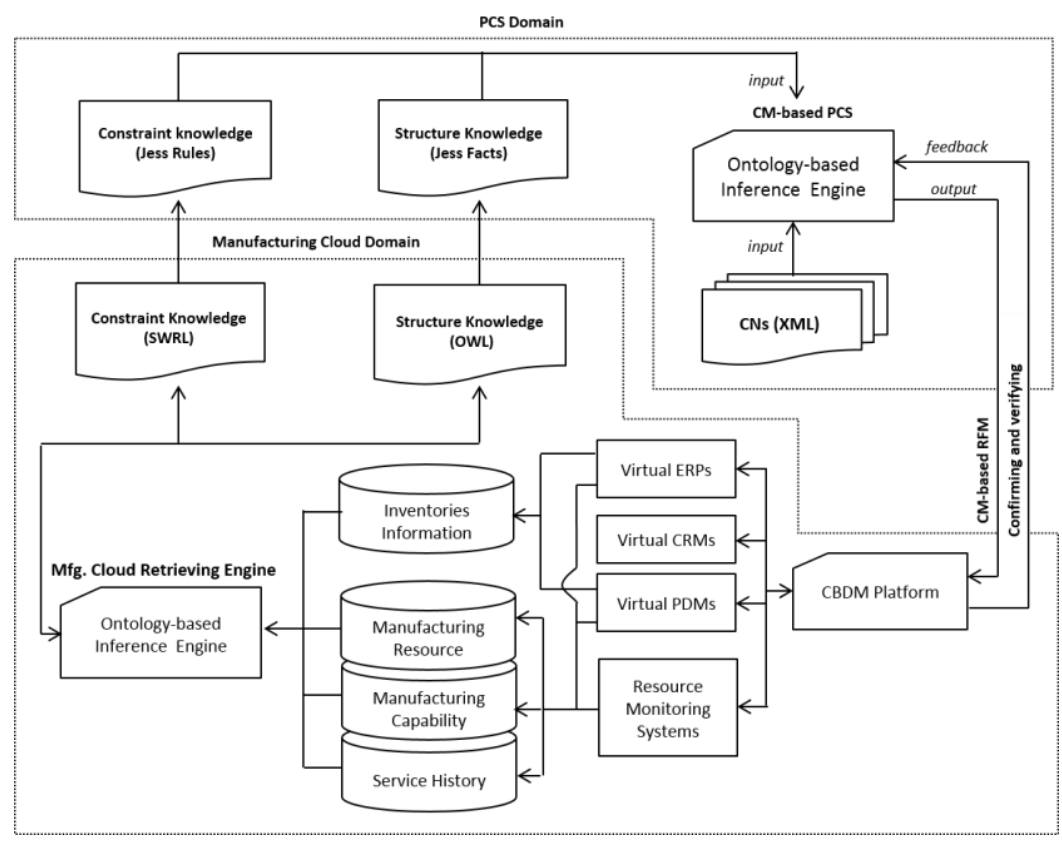

Fig. 2. Communication between PCS and Manufacturing Cloud

The communication mechanism, to address the first issue, proposed in this paper is shown as Fig. 2. According to the diagram, the interaction between PCS domain and 
Manufacturing domain mainly depend on the introducing of Manufacturing Cloud retrieving engine which is actually an ontology-based reasoning engine.

To address the second issue, a shared ontology for all service providers describing available product catalogues, inventories, manufacturing resources and manufacturing capability is proposed. In this way, supplementary configuration knowledge from cloud encoded in the format of OWL and SWRL could be transformed into Jess facts and rules for inference. The advantage of OWL-based configuration models is that the reuse of configuration models can be ensured since OWL, as an ontology language, supports knowledge reuse and sharing. The Jess rules mentioned in this diagram are developed by using the Extensible Style-sheet Language Transformations (XSLT) to perform the transformation from XML documents to Jess files.

After configuration reasoning, the CM-based PCS will generate not only a configuration but also a CM-based RFQ which may include request of procurement and outsourced manufacturing task enabled by manufacturing service provider via CBDM platform. This research utilise the interoperability of the STEP format to carry necessary information the RFM needed. In consideration of increasingly large product model, a new edition of Part 21 of ISO 10303 is adopted to archive and compress (in ZIP format) possible gigantic RFM information in configuration of complex ETO product. Part 21 is a specification for how to format entities describing product data [12]. It is a public domain file format for the geometry of assemblies and is widely used by industry to describe design and manufacturing specifications for airplanes, automobiles, ships, buildings and other products. This research archive RFM data by Part 21 Edition 3, so a parser capable of automatically interpreting configuration results into an EXPRESS schema is necessary to generate RFM.

\section{Case study}

To implement and validate methodologies proposed in this paper, this work chooses a local manufacturing firm, Compac Sorting Equipment (abbreviated as Compac in the following paper) to conduct a case study. Compac's headquarter is located in Auckland, New Zealand, and its branches and suppliers are around the world. Compac is a manufacturing firm producing sorting machines which are ETO products with high complexity. Typically, a sorting machine produced by Compac is a streamline machine system mainly including infeeding, sorting and grading, bagging and packing sub systems. To be specific, an infeed system is capable of tipping, washing and treatment of fruits, sorting and grading sector support sorting fruits by weight, colour, density, size, shape, surface defects and internal properties of fruits.

One thorny issue is configuration rules coding due to the complexity of their product. The company is working on developing a traditional rule-based configuration system. However, as above mentioned, knowledge reuse and sharing is essential in a configuration in cloud environment. This research therefore transforms their codes into the ontology model of product configuration and associated rules in parallel.

As above-mentioned methodologies, this research adopts ontology-based PCS to tackle configuration task, and the ontology model of the main component of sorting 
machine are built. The ontology model proposed is modularised according to the features of the sorting machine which typically consist of a sorter, washer and packer. The constraint knowledge is modularised as different cluster. The essential data for CMbased RFM is compressed as a ZIP archive.

As Fig. 3 shown, a configuration is generated according to CNs and cloud-sourced knowledge, then configuration result is compressed in accordance with P21 Edition 3.

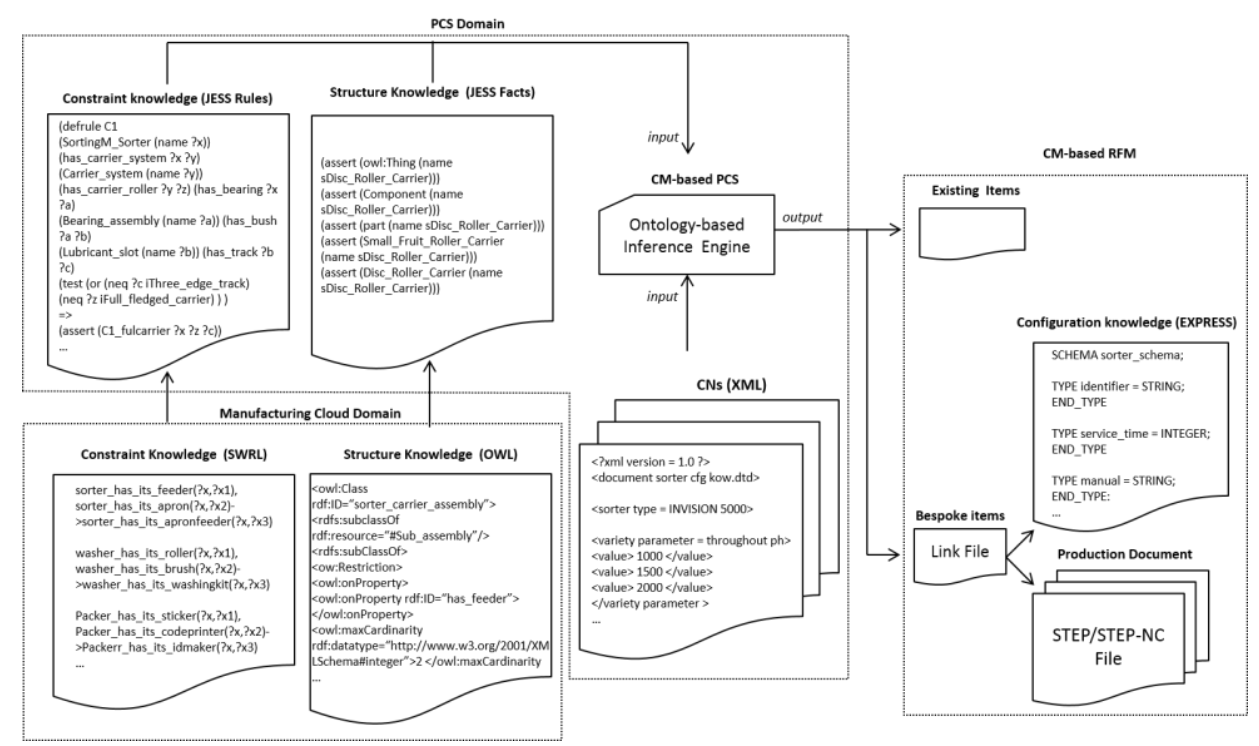

Fig. 3. Product configuration of sorting machine

This case study only establishes a prototype of product configuration system for cloud manufacturing. Due to the complexity of products, a complete PCS for a whole streamline equipment required more endeavour to achieve. Even so, the current prototype has demonstrated that such a methodology of developing CM-based PCS is practical and feasible.

\section{Conclusions}

This paper has first presented the survey on existing commercial cases of product configuration solutions and a literature review on technologies associated with PCS. The research concluded that even state-of-the-art product configuration tools are far from being satisfactory and the introduction of cloud manufacturing has the potential to enhance conventional PCS by boosting connectivity, enabling customisation freedom management and facilitating implementation with a low effort. To integrate Manufacturing Cloud with PCS, this paper proposed a methodology for implementing CMbased PCS. In order to utilise huge amount of cloud-sourced configuration knowledge, 
an ontology configuration model has been adopted to realise knowledge reuse and sharing in a ubiquitous cloud. The prototype of CM-based PCS proposed here is accessible to internal and external manufacturing resources via Manufacturing Cloud. Meanwhile, inclusive manufacturing services could enrich configuration options to some extent. Most importantly, an open architecture platform based on general ontology configuration model could be developed to reduce the cycle of PCS implementation. This research validates methodologies through a case study of complex ETO products.

The prototype of CM-based PCS described in this paper promises to transform existing PCSs into full-fledged cloud solution. Nevertheless, it has some limitations. For example, ontology models in this paper were built manually. This is because so far there has not been an effective way to generate ontology automatically. Additionally, further study has been planned to retrieve, filter and optimise available cloud services in complex cloud environment.

Acknowledgements. The work presented in this paper has been financially supported by the China Scholarship Council and the University of Auckland Joint Scholarship. The authors also wish to acknowledge the contribution from partner company, Compac.

\section{References}

1. Ferrer, G.: Open architecture, inventory pooling and maintenance modules. International Journal of Production Economics. 128(1), 393-403 (2010)

2. Zhou, F., Y. Ji, and R.J. Jiao: Affective and cognitive design for mass personalization: status and prospect. Journal of Intelligent Manufacturing. 24(5), 1047-1069 (2013)

3. $\mathrm{Xu}, \mathrm{X}$.: From cloud computing to cloud manufacturing. Robotics and computerintegrated manufacturing. 28(1), 75-86 (2012)

4. Li, B.-H., et al.: Cloud manufacturing: a new service-oriented networked manufacturing model. Computer Integrated Manufacturing Systems. 16(1), 1-7 (2010)

5. Wu, D., et al.: Enhancing the Product Realization Process with Cloud-Based Design and Manufacturing Systems. Journal of Computing and Information Science in Engineering. 13(4), 041004 (2013)

6. Yip, A.L.: A Product Configurator for Cloud Manufacturing. MSEC2013, vol. 1250, pp. V002T02A010. (2013)

7. $\mathrm{Lu}, \mathrm{Y} ., \mathrm{X} . \mathrm{Xu}$, and J. Xu: Development of a Hybrid Manufacturing Cloud. Journal of Manufacturing Systems. 33.4, 551-566 (2014)

8. McGuinness, D.L. and F. Van Harmelen: OWL web ontology language overview. W3C recommendation. 10(10), (2004)

9. Horrocks, I., et al.: SWRL: A semantic web rule language combining OWL and RuleML. W3C Member submission. 21, 79 (2004)

10. Friedman, E., Jess in action: rule-based systems in java. (2003)

11. Yang, D., M. Dong, and R. Miao: Development of a product configuration system with an ontology-based approach. Computer-Aided Design. 40(8): 863-878 (2008)

12. 10303-21, I., ISO 10303-21: Industrial automation systems and integration Product data representation and exchange - Part 21: Implementation methods: Clear text encoding of the exchange. (2002) 